\title{
DE SE INTERPRETATION AND VP ELLIPSIS
}

\author{
CHRISTOPHER TANCREDI \\ University of Tokyo
}

\begin{abstract}
This paper argues for a reanalysis of one of Fiengo and May's eliminative puzzles of ellipsis. Rather than take their judgments at face value, the judgments themselves are re-evaluated and the missing reading is found to be possible in certain discourse contexts. Additionally, the missing reading is shown to be possible in examples in which certain of the pronouns cannot be given a de se interpretation. After setting out a comprehensive paradigm of examples controlling for de se interpretation, I show how this paradigm follows from analyses of pronominal anaphora developed in Tancredi $(1997,2000)$.
\end{abstract}

Keywords: VP ellipsis, de se interpretation, eliminative puzzles, anaphora

\section{VP Ellipsis and the Semantics of Pronominal Anaphora}

Since Sag (1976a, b) and Williams (1977) drew attention to the restrictions on pronominal interpretation found in ellipsis environments, VP ellipsis has been an important probe into the syntax and semantics of pronouns. The need to account for their restricted ambiguity in these environments provided one of the most compelling pieces of evidence for distinguishing between a bound variable interpretation and a referential interpretation for pronouns, a distinction that has become commonplace in syntactic and semantic analyses of pronominal interpretation (cf. Reinhart (1985, 1986), Fiengo and May (1994), Higginbotham $(1980,1983)$ among many others). In addition to these interpretations, it has been forcefully argued in Castañeda (1966, 1967a, 1967b, 1968), Lewis (1979), Higginbotham (1991) and Tancredi (1997) that pronouns can receive a de se interpretation. The interaction between de se interpretation and VP ellipsis has been explored as well, by Chierchia (1989) among others. However, none of the analyses of the interaction between VP ellipsis and pronominal interpretation developed so far in the literature has been comprehensive in that they have not sufficiently laid out the complex behavior that arises when VP

English Linguistics 19: 2 (2002) 308-334 - 308 --

(C) 2002 by the English Linguistic Society of Japan 
ellipsis contains multiple occurrences of pronouns.

The most complete work in this area has been done by Fiengo and May (1994) (henceforth F\&M). As with much work on VP ellipsis, however, F\&M focus primarily on the denotation of the relevant pronouns, ignoring the de se/non-de se quality of the pronouns' interpretation. In this paper I will show that their failure to pay sufficient attention to de se interpretation has led them to a theory of pronominal interpretation which technically fails to account for their own intuitions, and is essentially uninsightful. I propose an alternative analysis of the ambiguities found in such cases of VP ellipsis which is based on the semantics of anaphora developed in Tancredi (1997) for de se interpretation and in Tancredi (2000) for non-de se interpretation. These analyses are non-index based analyses of anaphora and hence compatible with recent trends toward a minimalist syntax (cf. Chomsky (1995, 2000)).

\subsection{Sag's and Williams' Strict and Sloppy Identity Restrictions}

Sag (1976a, b) and Williams (1977) independently noticed that pronouns contained in ellipsis environments are restricted in their interpretation in a manner which depends on how the non-elided occurrence of the pronoun is interpreted, an observation extended to deaccenting environments in Tancredi (1992). To see this, consider the following example. (Small italics indicates deaccenting.)

(1) John thinks people like him. Bill does/thinks people like him too.

Two separate cases need to be considered: (i) the case in which him in the first sentence is taken to be semantically anaphoric (directly or indirectly) on John, and (ii) the case in which it is not. In case (i), it is observed that the second sentence is ambiguous between two readings. In the first reading, the strict reading, the referent of the pronoun remains unchanged in the deaccented VP, the second sentence being interpreted to mean that Bill thinks people like John. In the second reading, the sloppy reading, the pronoun is claimed to act like a variable in the deaccented VP bound by the NP Bill, with the second sentence thereby coming to mean that Bill thinks people like Bill. Missing is a third logical possibility in which the pronoun in the deaccented VP is interpreted as anaphoric on some other expression outside the sentence or is interpreted demonstratively. Case (ii) illustrates the converse restriction. If the pronoun in the antecedent clause is taken 
to refer to Sam, either demonstratively or anaphorically, then the corresponding pronoun in the deaccented VP must refer to Sam as well. Interpretation of this latter pronoun as referring to John, Bill or anyone else is impossible in this case.

Though different in detail, the analyses that Sag and Williams offer of this phenomenon are largely similar. Both require that the elided VP be semantically identical with an antecedent. The possibility of sloppy identity is allowed for by analyzing VPs semantically as lambda abstracts and allowing the lambda operator to bind not only a variable occupying the subject thematic role of the verb but also a pronoun interpreted as a variable within the VP. For (1) such a sloppy reading is generated by interpreting the two VPs as in (2a). A strict identity interpretation for (1) can be generated by interpreting the two VPs as in (2b) (case (i) above)) or by interpreting them as in (2c) (case (ii)).

(2) a. $\lambda x . x$ thinks people like $x$

b. $\lambda x . x$ thinks people like john

c. $\lambda x . x$ thinks people like sam

The need to account for both strict and sloppy identity interpretations for pronouns in VP ellipsis environments has been taken as evidence that pronouns are ambiguous, interpretable either as variables as in (2a) or as referring expressions as in (2b).

\subsection{Fiengo and May's Many Pronouns Puzzle}

Fiengo and May (1994) argue against the analyses of Sag and Williams on the grounds that these analyses fail to provide a solution to three puzzles. In this paper, I will only be concerned with one of these three, the many pronouns puzzle illustrated in (3) below.

(3) a. Max said he saw his mother and Oscar $\mathrm{did} /$ said he saw his mother too

b. Max claims he swore he was innocent and Oscar does/ claims he swore he was innocent too

$\mathrm{F} \& \mathrm{M}$ restrict their attention to interpretations of the sentences in which the pronouns in the first conjunct are intended as anaphorically related to Max. Holding this constant, Sag's and William's analyses each predict that these sentences should both be 4-ways ambiguous, i.e. that all logically possible combinations of strict and sloppy interpretations for the two VP-elided pronouns should be attested. F\&M, however, claim that only three such interpretations are realized: an across-the-board strict reading, an across-the-board sloppy reading, and a mixed reading 
in which the first elided pronoun is interpreted sloppily and the second strictly. The remaining combination in which the first elided pronoun is interpreted strictly and the second sloppily is claimed to be impossible.

To account for these claims, F\&M propose to complicate the syntax of pronouns. Under their analysis, all pronouns bear an index in the syntax. This index can be one of two distinct types - an $\alpha$-occurrence or a $\beta$-occurrence. An expression bearing an $\alpha$-occurrence of an index is independently valuated. Formally, this amounts to the expression being directly associated with a position in a $\sigma$-sequence which is itself associated with either an individual or a variable in the semantics. An expression bearing a $\beta$-occurrence of an index, in contrast, is dependently valuated, which means that it can only be given a semantic value (=be associated with an individual or variable via a position in a $\sigma$-sequence) by picking this value up from some antecedent expression occurring in the same sentence. To account for the restrictions on pronominal interpretation found to obtain in VP ellipsis environments, $\mathrm{F} \& \mathrm{M}$ propose that VP ellipsis requires the elided VP to be a reconstruction of its antecedent. Two VPs that are absolutely identical count as reconstructions of one another. However, given F\&M's assumption that a pronoun's indexical value strictly determines its interpretation, an elided VP that is syntactically identical with its antecedent will at most give rise to a strict identity interpretation of any pronouns contained therein. To account for the possibility of both strict and non-strict interpretations of VP-elided pronouns, F\&M take two VPs to be reconstructions of one another if everything other than the pronouns in the two VPs is identical and the pronouns either (i) occur in identical indexical dependencies, or (ii) have the same indexical value.

F\&M's analysis of the many pronouns puzzle illustrated with (3) depends crucially on their analysis of indexical dependencies (IDs). An ID is a triple consisting of a sequence of elements $S=\left(\mathrm{c}^{\alpha}{ }_{1}, \mathrm{c}^{\beta_{2}}, \ldots, \mathrm{c}^{\beta}{ }_{\mathrm{n}}\right)$, an index $I$, and a partial factorization $F=<$ category $_{i}$, category $y_{i+1}, \ldots$, categor $y_{i+n}>$ of contiguous factors of a structural description. The sequence of elements must contain only expressions bearing the index I, including exactly one element bearing an $\alpha$-occurrence, and one or more bearing a $\beta$-occurrence. Additionally, $F$ must be the smallest factorization which contains each of the elements in S. ${ }^{1}$

1 See F\&M pp. 51 ff. for finer details. 
The analysis just given is intended to account for the observed interpretations of (3a) as follows. The four logically possible combinations of strict and sloppy readings for the pronouns in (3a) can be derived from the following indexings:

(4) a. Max ${ }_{1}$ claims he ${ }^{\alpha}{ }_{1}$ swore he ${ }^{\alpha}{ }_{1}$ was innocent and Oscar ${ }_{2}$ claims he ${ }_{1}^{\alpha}$ swore $h e^{\alpha}{ }_{1}$ was innocent

b. $\operatorname{Max}_{1} \ldots \mathrm{he}^{\beta_{1}} \ldots \mathrm{he}^{\beta_{1}} \ldots$ and $\operatorname{Oscar}_{2} \ldots \mathrm{he}^{\beta_{2}} \ldots \mathrm{he}^{\beta_{2}} \ldots$

c. $\operatorname{Max}_{1} \ldots \mathrm{he}^{\beta_{1}}{ }_{1} \ldots \mathrm{he}^{\alpha}{ }_{1} \ldots$ and $\operatorname{Oscar}_{2} \ldots \mathrm{he}^{\beta_{2}} \ldots \mathrm{he}^{\alpha}{ }_{1} \ldots$

d. $\operatorname{Max}_{1} \ldots$ he $^{\alpha}{ }_{1} \ldots$ he $^{\beta}{ }_{1} \ldots$ and $\operatorname{Oscar}_{2} \ldots$ he $^{\alpha}{ }_{1} \ldots$ he ${ }^{\beta}{ }_{2} \ldots$

In the first 3 cases, the elided VP counts as a reconstruction of the VP in the first conjunct. In all 3 cases, the non-indexed material is identical. Further, in (4a), the indexed expressions in the VPs all bear occurrences of identical indices. In (4b), the pronouns in the two conjuncts are in identical ID relations. In the technical jargon that F\&M employ, the dependencies $-<\left(\operatorname{Max}^{\alpha}\right.$, he $\left.{ }^{\beta}, h^{\beta}\right), 1,<\mathrm{NP}, \mathrm{V}, \mathrm{NP}, \mathrm{V}$, $\mathrm{NP}>>$ and $<\left(\mathrm{Oscar}^{\alpha}\right.$, he ${ }^{\beta}$, he $\left.{ }^{\beta}\right), 2,<\mathrm{NP}, \mathrm{V}, \mathrm{NP}, \mathrm{V}, \mathrm{NP}>>-$ are $i$ copies of one another. Finally, in (4c), the pronouns with $\alpha$-occurrences bear the same index, while the pronouns with $\beta$-occurrences occur in identical IDs $-<\left(\operatorname{Max}^{\alpha}, h^{\beta}\right), 1,<\mathrm{NP}, \mathrm{V}, \mathrm{NP}>>$ and $<\left(\right.$ Oscar $^{\alpha}$, he $\left.^{\beta}\right), 2,<\mathrm{NP}, \mathrm{V}, \mathrm{NP}>>$. In (4d), on the other hand, the pronouns with $\beta$-occurrences neither have the same indexical value nor occur in identical dependencies. The pronoun in the elided clause is realized in the ID $<\left(\mathrm{Oscar}^{\alpha}\right.$, he $\left.^{\beta}\right), 2,<\mathrm{NP}, \mathrm{V}, \mathrm{NP}, \mathrm{V}, \mathrm{NP}>>$, while that in the antecedent clause is realized in the dependency $<\left(\mathrm{he}^{\alpha}, \mathrm{he}^{\beta}\right)$, $1,<\mathrm{NP}, \mathrm{V}, \mathrm{NP}>>$. The fact that these pronouns are not co-indexed and also fail to be realized in identical dependencies means that they are not reconstructions of one another, and hence that the VPs that contain them are also not reconstructions of one another. It follows that this indexing is not a possible one for the VP ellipsis example in (3a). F\&M take this to explain the claimed absence of the fourth logically possible reading for this example.

It is worth examining the analysis that leads to this conclusion in some detail. The analysis contains three important stipulations: (i) the sequence of elements $\mathrm{c}^{\alpha}{ }_{1}, \mathrm{c}^{\beta}{ }_{2}, \ldots, \mathrm{c}^{\beta}{ }_{n}$ that constitutes the first part of a dependency ID must contain exactly one expression with an $\alpha$-occurrence; (ii) the factorization constituting the third part of ID must contain all and only the categories in some structural description (SD) starting from that of the first element, $\mathrm{c}^{\alpha}{ }_{1}$, and ending with that of the last, $\mathrm{c}^{3}$; and (iii) every category in the factorization that bears the in- 
dex that constitutes the second part of ID must occur in the sequence $\mathrm{c}^{\alpha}{ }_{1}, \mathrm{c}^{\beta}{ }_{2}, \ldots, \mathrm{c}^{\beta}{ }_{\mathrm{n}}$. These stipulations make it impossible to analyze he ${ }^{\beta}{ }_{1}$ in (4d) as realized in a dependency headed by $\operatorname{Max}^{\alpha}{ }_{1}$. Any factorization of the SD that contains (the syntactic categories of) these two expressions will also contain an additional expression bearing an $\alpha$ occurrence of the index 1 , namely the expression he ${ }^{\alpha}{ }_{1}$, and yet this latter expression cannot be added to a sequence containing the former two in an ID since the resulting sequence would then contain two $\alpha$ occurrences, in violation of the stipulation in (i). This result can be seen as a specific instance of a more general restriction on dependencies that will be of great importance below: for any categories $a, b$ and $c$ such that $a$ c-commands $b$ and $b$ c-commands $c, a$ and $c$ cannot be categories in the factorization of an ID unless $b$ is a category in that factorization as well.

\section{Problems with F\&M's Analysis}

Under F\&M's analysis, c-command relations among pronouns in a dependency are central to explaining the alleged absence of the strictsloppy reading in (3). If $a$ c-commands $b$ which in turn c-commands $c$, and if $a$ and $b$ both bear $\alpha$-occurrences of the same index, then it is impossible for $c$ to be realized in a dependency with $a$. Since in the typical case an $\alpha$-occurrence on $b$ is necessary for generating a strict interpretation of $b, \mathrm{~F} \& \mathrm{M}$ conclude that a strict-sloppy reading of $b-c$ is impossible whenever these c-command relations obtain. The sentences in (5), however, show that F\&M's explanation cannot be correct.

(5) a. Max claims that he is near his house. Oscar does/claims that he is near his house too.

b. Max thinks he deserves the award he got. Oscar does/ thinks he deserves the award he got too.

c. Max wonders how he ruined his shirt. Oscar does/wonders how he ruined his shirt too.

Taking the pronouns in the first sentences in $(5 \mathrm{a}-\mathrm{c})$ to refer ultimately to Max, the second sentences of these examples are each 4-ways ambiguous. Thus (5a), for example, can mean among other things that Oscar claims that Max is near Oscar's house, the strict-sloppy pattern that $F \& M$ found to be unavailable in (3). This interpretation can be facilitated by an appropriate context, such as that given in (6), though the context is not strictly speaking necessary. 
(6) Max and Oscar disagree about where Max is. Max/He claims that he is near his house, and Oscar does too, but Max's house and Oscar's house are miles apart.

This same strict-sloppy pattern surfaces equally in $(5 b, c)$ as well. In all of the examples in (5), however, the matrix subject of the first sentence asymmetrically c-commands the first embedded pronoun, which in turn asymmetrically c-commands the second, just as in (3). Since the structural differences between the examples in (3) and (5) are irrelevant to the predictions of F\&M's analysis, the availability of an extra reading for the latter examples shows that this analysis cannot be correct.

Matters are even worse for $F \& M$ when one embeds their original examples into a context which controls for de se interpretations, for here as well one finds that the alleged missing readings become readily available. In particular, a context which requires the first pronoun to be interpreted non-de se and favors a de se interpretation of the second pronoun will make a mixed strict-sloppy reading possible. To see this consider the occurrence of (3a) in the following.

( 7 ) Who do people think that Max saw?

Max said he saw his mother, and Oscar did too.

If the occurrence of he in (7) is taken to be anaphoric on the occurrence of Max in the question, it can more or less naturally be given a non-de se interpretation while the second pronoun his is interpreted de se with respect to the occurrence of Max in the answer. Given this pattern of interpretation for the pronouns in Max said he saw his mother, a strict-sloppy reading for the VP ellipsis sentence Oscar did too is straightforwardly available.

Given the unwieldy complexity of F\&M's analysis, it is not surprising that it turns out to do things that they themselves claim it cannot. In fact, the analysis is capable of generating the very strict-sloppy pattern of interpretation that they claim is unavailable for sentences like those in (3), contrary to their own claims. This interpretation is generated by the indexing in (8).

(8) $\operatorname{Max}_{1} \ldots$ he $^{\beta_{1}} \ldots$ he $^{\beta_{1}} \ldots$ and $\operatorname{Oscar}_{2} \ldots$ he $^{\beta_{1}} \ldots$ he $^{\beta_{2}} \ldots$ All of the expressions in (8) have the same indexical values as (4d). The two examples differ only in that the $\alpha$-occurrences of pronouns from (4d) are changed to $\beta$-occurrences in (8). This minimal change still satisfies all restrictions on $\alpha$-occurrences and $\beta$-occurrences of indices. In particular, all of the $\beta$-occurrences are contained within IDs 
headed by an $\alpha$-occurrence. More significantly for our present purposes, the matrix VP of the second conjunct counts as a reconstruction of that of the first conjunct. $\mathrm{He}^{\beta_{2}}{ }_{2}$ is contained in an identical ID as the corresponding occurrence of he ${ }^{\beta}{ }_{1}$ in the antecedent clause, the latter ID headed this time by $\mathrm{Max}_{1}$. Furthermore, he ${ }^{\beta_{1}}$ in the VP ellipsis site has the same indexical value as the corresponding occurrence of he $^{3}{ }_{1}$ in the antecedent clause. ${ }^{2}$

Though one might be tempted at first to take this to be an unintended positive result of their analysis, that temptation is soon dashed. The unintended success of their analysis depends on the fact that the examples in (3) are single sentences. Only in this case will the VPelided occurrence of he ${ }^{\beta}$ be contained in a legitimate ID since IDs do not obtain across sentences. The problem is that the same strict-sloppy interpretation found by embedding (3a) in the context in (7) arises equally for the non-conjoined counterpart of (3a) given in (9).

(9) Max said he saw his mother. Oscar $\mathrm{did} /$ said he saw his mother too.

Similar comments apply to the sentences in (5). Thus F\&M's analysis suffers from four major defects. First, it makes an incorrect claim about the data. Second, the actual predictions of the theory are distinct from the predictions they claim the theory makes. Third, the actual predictions are not consistent with the claimed data. Fourth, neither the actual predictions nor the claimed predictions are consistent with the actual data. The theory is thus wrong by its own lights, as well as by anybody else's.

\section{Background to an Analysis}

As the above discussion suggests, the possibility of generating a particular pattern of strict and/or sloppy identity interpretations for pronouns in VP ellipsis contexts depends in part on how the pronouns are

2 Note that the first ID will contain 3 expressions $\left(\operatorname{Max}_{1}, h^{\beta_{1}}{ }_{1}\right.$, he $\left.{ }^{\beta_{1}}{ }_{1}\right)$ while the second ID will only contain $2\left(\mathrm{Oscar}_{2}, \mathrm{he}^{\beta_{2}}\right)$. This difference, however, cannot be used to avoid the predictions made in the text without making it impossible to account for the strict/sloppy ambiguity in examples like (i).

( i ) Max said he loves his mother and Bill said you do/love his mother. 
interpreted in the antecedent VP. In particular, whether the antecedent pronouns are anaphoric and if so whether they are de se or non-de se can affect the possible interpretations of the elided pronouns. In order to give an explanation of the phenomenon hinted at in F\&M's many pronouns puzzle, it is first necessary to set up a complete paradigm of examples which controls for the type of interpretation of each of the pronouns. Second, it is necessary to spell out the semantics of anaphoric interpretation, both de se and non-de se. Once this is accomplished, we can turn to the task of accounting for the full range of readings available in VP ellipsis environments.

\subsection{The Paradigm}

Given the complexity of the data, it will be useful to break the paradigm down into two separate parts: one for VP ellipsis examples containing a single elided pronoun, and one for cases containing two elided pronouns. Though I give a complete paradigm in the former case, to simplify the discussion I give only a pared down version of the paradigm in the latter case, eliminating from discussion cases which are straightforward but do not involve anaphoric interpretation as well as cases in which intuitions are insufficiently precise to make use of.

\subsubsection{Single VP-Elided Pronoun}

The first set of data contains a single pronoun in a VP ellipsis environment, such as (1), repeated here for ease of reference.

(1) John thinks people like him. Bill does/thinks people like him too.

The pronoun him in the first sentence of this example can be given at least three distinct types of interpretation: it can be a demonstrative pronoun, it can be anaphoric but non-de se, or it can be $d e s e .^{3}$ The first case occurs when him is accompanied by an act of ostension, and gives rise to only a strict identity reading of the VP-elided pronoun. The second case includes sub-cases of cross-sentential anaphora and of intra-sentential anaphora. The former can be illustrated by taking (1) as a continuation of a discourse initiated with the question What do

\footnotetext{
3 I assume that all instances of de se interpreted pronouns are indirectly anaphoric. Cf. footnote 9 for clarification.
} 
people think about John? In this case, him is most naturally interpreted as anaphoric on the occurrence of John in the question, and when so construed gives rise exclusively to a strict identity reading of the VP-elided pronoun. The latter sub-case of non-de se anaphoric interpretation is that best brought out by sentences about amnesics like those discussed by Higginbotham (1991) based on work by Castañeda (1966, 1967a, b, 1968). Consider an amnesia ward in a hospital, for example, in which for therapy all patients are given stories about their own pasts to read. With this as background, imagine a doctor uttering (10) about two patients, John and Bill.

(10) Based on what they read, John thinks people like him, and Bill does/thinks people like him too, (though neither of them consider THEMSELVES to be liked)

The sentence is to be taken as saying about John that he thinks that people like a certain person he read about, who happens (unbeknownst to him) to be John himself. Here, a strict reading for the VP-elided pronoun is unproblematic. A sloppy reading, in contrast, is more difficult. However, the difficulty involved would appear to stem at least in part from the fact that a de se interpretation of the pronoun in the antecedent VP is possible. If we consider a case in which a de se interpretation is impossible for that pronoun, as in (11), ${ }^{4}$ we find that a sloppy identity interpretation becomes very natural.

(11) John has an immune system which protects him from disease. Bill does too.

The final case to consider is that in which the first occurrence of the pronoun him in (1) is given a de se interpretation. This can be brought out by taking (1) to occur in the context of the sentence John is/John and Bill are so vain. ${ }^{5}$ In this case, both a strict and a sloppy interpretation of the elided pronoun appear to be equally possible. These results are summarized in the table below.

4 I owe this example to David Lewis (personal communication).

5 To make the sentences more natural, it is in some cases better to change the occurrence of the name John in (1) to the pronoun $\mathrm{He}$, and to thereby make the link with the preceding context more explicit. I ignore these details here. 


\begin{tabular}{|c|c|c|c|c|}
\hline \multirow[b]{3}{*}{$\begin{array}{c}\text { Interpretation } \\
\text { of elided } \\
\text { pronoun }\end{array}$} & \multicolumn{4}{|c|}{ Interpretation of first pronoun in (1) } \\
\hline & \multirow[b]{2}{*}{ Demonstrative } & \multicolumn{2}{|c|}{ Anaphoric non-de se } & \multirow[b]{2}{*}{$\begin{array}{c}\text { Anaphoric } \\
\text { de se }\end{array}$} \\
\hline & & $\begin{array}{c}\text { cross- } \\
\text { sentential } \\
\text { anaphoric }\end{array}$ & $\begin{array}{l}\text { intra-sentential } \\
\text { anaphoric }\end{array}$ & \\
\hline strict & ok & ok & ok & ok \\
\hline sloppy & * & $*$ & $\begin{array}{l}\text { ok (no de se potential) } \\
{\left[?^{*}(\text { potential de se)] }\right.}\end{array}$ & ok \\
\hline
\end{tabular}

TABLE 1: Correlation between strict/sloppy identity and type of interpretation of the antecedent pronoun in VP ellipsis environments containing a single pronoun. (Brackets [] indicate that the relevant reading is difficult to isolate.)

\subsubsection{Multiple VP-Elided Pronouns}

We are now ready to turn to cases in which the antecedent VP and the elided VP each contain two pronouns. Consider first the case of (3b) (=Max claims he swore he was innocent, and Oscar claims he swore he was innocent too). Each of the two pronouns can potentially be interpreted in either of the four different ways considered above. In addition, the second pronoun has two distinct ways of being interpreted de se-one via the matrix subject (=matrix de se) and one via the higher of the embedded subjects (=embedded $d e s e$ ). There are thus a total of 20 potential combinations to check for ambiguity with respect to strict and sloppy readings. To keep things manageable, I ignore demonstratively used pronouns, which uniformly give rise to only strict identity interpretations. I also ignore intra-sentential non-de se anaphora, since the judgments in these cases are extremely difficult to come by and are not sufficiently clear to base a theory on. The remaining combinations give rise to the following possibilities for strict and sloppy identity interpretations: 


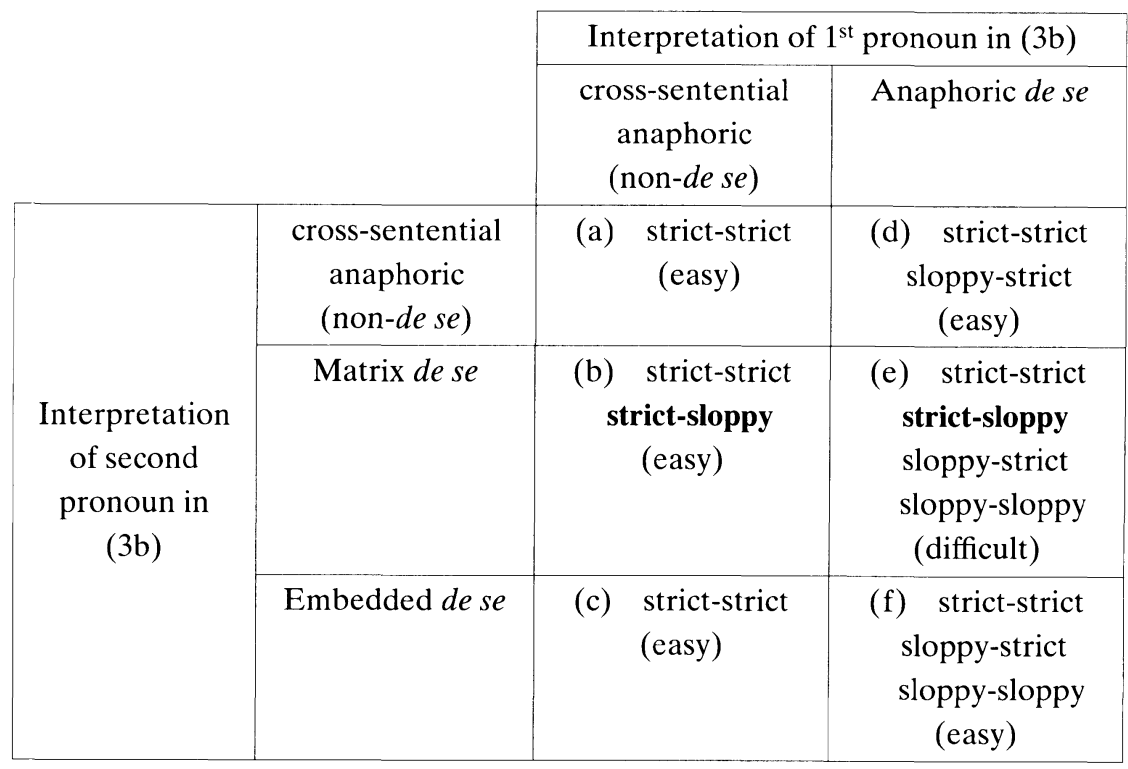

TABLE 2: Correlation between strict/sloppy identity and type of interpretation of the antecedent pronouns in (3b). (easy/difficult refers to the degree of difficulty in isolating the relevant combination of de se and/or non-de se anaphoric interpretations for the pronouns in the antecedent VP given an appropriate context.)

Contexts for obtaining the readings in each of the cells in Table 2 are given below:

(12) a. What did Max say about Max at the trial yesterday?

b-c. What did Max do at the trial yesterday?

d. What did people say about Max at the trial yesterday?

e-f. What happened at the trial yesterday?

As can be seen in Table 2, all combinations of strict and sloppy identity interpretations can be obtained for the VP ellipsis sentences. In particular, the strict-sloppy interpretation can be obtained in either of two ways. The first way is by analyzing the first pronoun in the antecedent VP as anaphoric on a sentence-external occurrence of Max and interpreting the second pronoun as de se via the matrix subject. This is relatively easy to do, though not a natural way to interpret the sentence out of the blue. The second way is to analyze both pronouns as de se via the matrix subject. This is very difficult to do, and probably the least natural way of understanding the first sentence of $(3 \mathrm{~b})$. None of 
the other ways of interpreting the first sentence of (3b) give rise to a strict-sloppy reading.

The last set of data to look at is the case of (5a) (=Max claims that he is near his house. Oscar does/claims that he is near his house too.), where a strict-sloppy interpretation is relatively easy to come by. This example differs from the examples in (3) in that the only way of giving the second pronoun in (5a) a de se interpretation is via the matrix subject. No psychological attitude is attributed to the embedded subject, making an embedded de se interpretation impossible. The different combinations of strict and sloppy interpretations for the pronouns in this example are given in Table 3.

\begin{tabular}{|c|c|c|c|}
\hline & \multicolumn{2}{|c|}{ Interpretation of first pronoun in (5a) } \\
\hline & & $\begin{array}{l}\text { cross-sentential } \\
\text { anaphoric } \\
\text { (non-de se) }\end{array}$ & Anaphoric de se \\
\hline \multirow{3}{*}{$\begin{array}{l}\text { Interpretation } \\
\text { of second } \\
\text { pronoun in } \\
(5 \mathrm{a})\end{array}$} & $\begin{array}{c}\text { cross-sentential } \\
\text { anaphoric } \\
\text { (non-de se) }\end{array}$ & $\begin{array}{l}\text { (a) strict-strict } \\
\text { (easy) }\end{array}$ & $\begin{array}{l}\text { (d) } \text { strict-strict } \\
\text { sloppy-strict } \\
\text { (easy) }\end{array}$ \\
\hline & Matrix de se & $\begin{array}{l}\text { (b) strict-strict } \\
\text { strict-sloppy } \\
\text { (easy) }\end{array}$ & $\begin{array}{l}\text { (e) strict-strict } \\
\text { strict-sloppy } \\
\text { sloppy-strict } \\
\text { sloppy-sloppy } \\
\text { (easy) }\end{array}$ \\
\hline & Embedded de se & (c) non-existent & (f) non-existent \\
\hline
\end{tabular}

TABLE 3: Correlation between strict/sloppy identity and types of interpretation of the antecedent pronouns in (5a).

Contexts for obtaining the readings in Table 3 are given below:

(13) a. Where is Max with respect to Max's house?

b. Where is Max?

c. Who is near Max's house?

d. Where is everybody?

Note that except for the non-existence of an embedded de se interpretation for the second pronoun, the strict-sloppy combinations available in (5a) are exactly identical to those available in (3b). All that differs is the relative ease with which the various combinations of de se and/or anaphoric non-de se readings can be obtained for the first sen- 
tences. In particular, the reading represented by cell (e) of Table 2 was seen to be difficult to obtain for the antecedent sentence, while the corresponding interpretation in Table 3 is relatively easy to obtain.

\subsection{The Semantics of Anaphoric Interpretation}

As the above discussion suggests, I take cross-sentential anaphora and de se interpretation to be semantically distinct and distinguishable processes. I have spelled out the basic analysis I assume of the former in Tancredi (2000), and of the latter in Tancredi (1997). For the motivation behind these two analyses I refer the reader to the abovementioned papers. Here I simply outline the results from those papers that I will make use of here. In the case of de se anaphora I will modify and extend the analysis slightly.

\subsubsection{Cross-sentential Anaphora}

I take cross-sentential pronominal anaphora to be an instance of a pure individual interpretation of a pronoun. Such pronouns are assumed to be unindexed in the syntax, and to pick up as their interpretation an individual (or individual variable) from the Conceptual Structure (CS) representation of a given discourse situation. Anaphoric interpretation is resolved, then, in the mapping from Logical Form (LF) to CS. Importantly, though this mapping can give rise to a bound variable interpretation for a pronoun, it does not give rise to de $s e$ interpretation.

\subsubsection{De Se Anaphora}

There are two basic ingredients to de se interpretation. The first is reference to an individual via a thought attributed to that individual. In Tancredi (1997) this idea is implemented by analyzing de se pronouns semantically as definite descriptions whose interpretation is equivalent to "the individual $\mathrm{x}$ who has thought $\mathrm{t}$. " 6 This by itself will secure reference whenever $t$ is given an appropriate value. However, the semantic interpretation of the pronoun alone is not sufficient to

6 For problems with this analysis, cf. Oshima (2001). The problems raised there do not directly affect the applications of the analysis in this paper, though they do show up a need to re-examine the foundations of the analysis. 
generate a de se interpretation. In addition, the pronoun must be used to characterize a thought attributed to the person to whom the pronoun refers. To illustrate, consider the following sentence, with the pronoun interpreted as indicated above:

(14) John thinks he is brilliant.

Suppose first that the thought $t$ contained in the interpretation of the pronoun is attributed to Bill. In this case, (14) will amount to saying that John thinks Bill is brilliant, since Bill is the individual $\mathrm{x}$ who has thought $t$. There is nothing special about such a use of he in (14) because there is no special relation that John stands in to a thought of Bill's. The pronoun in this use clearly does not generate a de se interpretation, though this constitutes a perfectly legitimate form of reference.

If the thought $t$ contained in the interpretation of the pronoun in (14) is a thought attributed to John, things are different. Since John identifies his own thoughts in a very special way - in the words of Evans (1982) in a way that is immune to error through misidentification - the individual picked out as having those thoughts stands out in John's mind as distinct from any other individual having thoughts, for that is the individual who has the very thoughts that John himself directly experiences. If the pronoun refers by way of the thought $t$ attributed to John, then, it will be given a special interpretation provided that it is used to characterize a thought $T$ attributed to John. The thought characterized $(=T)$ may, but need not, be identical to the thought $t$ used to identify the individual that John is claimed to be thinking about. I refer to the special case in which thoughts $t$ and $T$ are the same token thought as semantic de se, and assume that this case comes about via the interpretation of $t$ as semantically anaphoric on $T$. The case in which the two thoughts are distinct token thoughts both attributed to the same person I refer to as pragmatic de se, which I assume comes about via the pragmatic identification of $t$ as an independent thought attributed to the same person that $T$ is attributed to. ${ }^{7}$

7 Formally, I take both instances of anaphora resolution to be identical. In both cases, $t$ picks up its reference from among those thoughts represented at CS. The labels "semantic" and "pragmatic" are meant as mnemonic devices that help to identify where the CS represented thought is taken to originate from. 
4. Interaction of Pronominal Interpretation and VP Ellipsis/Deaccenting

We are now ready to turn to an explanation of the patterns of strict and sloppy identity found in the VP ellipsis examples in (3) and (5). Recall that F\&M claimed with respect to the former examples that a strict-sloppy interpretation is impossible, and proposed to exclude this possibility by complicating the syntax of coreference and dependency. I have claimed in contrast that the reading they claim to be absent is in fact available. This reading is difficult to obtain in the sentences in (3) if these sentences are taken to occur out of the blue, but much easier to obtain when these sentences are embedded in a context which allows for the pronouns to take an extra-sentential antecedent. This reading is also readily available in the examples in (5) which are phrase-structurally identical with the sentences in (3) in all respects relevant to F\&M's analysis. This leaves me with two tasks. The first is to show how the analyses of anaphoric interpretation given in section 3 can combine with suitable restrictions on VP ellipsis to predict the full range of interpretations found. The second is to explain why it is that some of those readings are more difficult to obtain than others. I tackle these two tasks in the following sections.

\subsection{The Analysis}

Given the analyses of anaphoric interpretation of pronouns assumed, generating all and only the readings found in Tables 1-3 can be done by adopting a very simple restriction on VP ellipsis: identity of interpretation at CS with an antecedent VP. ${ }^{8}$ Appeal to an otherwise unmotivated notion of reconstruction is unnecessary. To see why, consider each of the three relevant ways that a pronoun in the antecedent VP can be interpreted: (cross-sententially anaphoric) non-de se, semantic de se, and pragmatic de se. The first results in a pure individual interpretation for such a pronoun. For a VP-elided pronoun to have the

8 In the case of VP ellipsis, this could as easily be analyzed as another instance of anaphoric interpretation, this time of a dummy verbal element rather than of a pronoun. Since this same analysis cannot readily be carried over to the case of deaccented VPs, I do not pursue it here. 
same interpretation at $\mathrm{CS}$ as such a pronoun requires that the two pronouns refer to the same individual. In this case only a strict identity interpretation for the VP-elided pronoun can be generated. This conforms with the observations summarized in Tables 1-3.

Second, consider the case of semantic de se interpretation. This is the case in which the thought contained in a de se pronoun is identified with a thought attributed by a predicate to what in an extended sense can be called the antecedent of the pronoun. ${ }^{9}$ If we make the natural assumption that a thought attributed by a predicate is existentially quantified within the lexical semantics of the predicate, then in cases of semantic de se interpretation, both the thought contributed by the predicate and that contributed by the pronoun will be bound by the same existential quantifier within the VP headed by the predicate. To see this, consider the case of (15a) with the simplified semantics given in $(15 b-d) .{ }^{10}$

(15) a. John thinks that he won

b. $\llbracket$ think $\rrbracket=\lambda$ p. $\lambda x . \exists T[x$ has (thought token) $\mathrm{T}$ and $[\forall \mathrm{w}$ :

$\mathrm{T}$ is true in $\mathrm{w}](\mathrm{p}(\mathrm{w}))=1]$

c. $\llbracket$ that he won $\rrbracket=\lambda w$.the individual with thought $t$ won in $\mathrm{w}$

d. 【think that he won $\rrbracket=\lambda \mathrm{x} \cdot \exists \mathrm{T}[\mathrm{x}$ has (thought token) $\mathrm{T}$ and $[\forall \mathrm{w}$ : $\mathrm{T}$ is true in $\mathrm{w}]$ (the individual with thought $T$ won in $w)]$

The thought contained in the pronoun is interpreted here as anaphoric on $\mathrm{T}$ and hence bound by the existential quantifier $\exists \mathrm{T}$. For an elided VP to have an identical interpretation at CS, it too must contain an existential quantifier binding thoughts in identical positions. If the

9 Strictly speaking, de se pronouns do not have antecedents, i.e. expressions they are dependent on which are interpreted identically. The anaphora inherent in the interpretation of de se pronouns is anaphoric interpretation of a thought, not of an individual. Hence literally speaking, it is only the thought that has an antecedent, not the pronoun as a whole. For ease of exposition, however, I will often speak loosely of a referring expression as being the antecedent of a de se pronoun, though this should never be taken literally.

10 I realize that this glosses over several important issues in the semantics of propositions, though because of space limitations I leave these issues unaddressed in this paper. 
thought contributed by the predicate is then attributed to a distinct subject in the VP ellipsis sentence, the VP-elided pronoun will thereby come to refer to the subject of the VP ellipsis sentence, giving rise to a sloppy identity interpretation. Sloppy identity is thus predicted to be compatible with de se interpretation of a pronoun in an antecedent VP. Again this is compatible with the observations made in Tables 1-3.

Finally, consider the case of pragmatic de se. In this case, the thought contained within a de se pronoun and that attributed by a predicate to the "antecedent" of the pronoun are distinct thoughts independently attributed to the same individual. The former is not bound by the existential quantifier that binds the latter. For the matrix VP of the sentence in (15a), this would result in the interpretation given in (16), where $\mathbf{t}$ is anaphoric on a thought attributed to John that is not contributed by the occurrence of the predicate think in this sentence, and so $\mathbf{t} \neq \mathrm{T}$.

(16) 【think that he won $\rrbracket=\lambda \mathrm{x} . \exists \mathrm{T}[\mathrm{x}$ has (thought token) $\mathrm{T}$ and $[\forall \mathrm{w}: \mathrm{T}$ is true in $\mathrm{w}$ ] (the individual with thought $\mathbf{t}$ won in w)]

If a VP such as that in (16) serves as the antecedent to VP ellipsis, then the elided VP will have to contain a pronoun having the same interpretation as the de se pronoun in the antecedent VP. If the pronoun in the antecedent VP is interpreted as "the individual $\mathrm{x}$ who has thought t," then the pronoun in the elided VP must be so interpreted as well. While the former will be interpreted de se, however, in the cases of VP ellipsis considered in this paper the latter will not be. This is so for a simple reason: while the antecedent VP attributes a thought to the antecedent of the pronoun contained in that VP, the same does not hold for the elided VP. This means that a de se interpretation of a pronoun in an antecedent VP is compatible with a strict identity, non-de se interpretation in an elided VP. Combined with the predictions for semantic de se pronouns, it thus follows that a de se interpreted pronoun in an antecedent VP can give rise to either a strict or a sloppy identity interpretation in an elided VP.

Inspection of the examples in Tables 1-3 will show that the predictions made by the above theory are exactly borne out. I illustrate for the one case which is somewhat less than straightforward, that of cell (f) from Table 2. This is the cell corresponding to a local de se interpretation of both occurrences of the pronoun he in the first sentence in example (3b) (=Max claims he swore he is innocent. Oscar does too.) 
The first of these pronouns can give rise to either a strict or a sloppy identity interpretation in the VP ellipsis sentence as just described above depending on whether it is interpreted as semantically de se or pragmatically de se. The second of these pronouns can again be either semantically de se or pragmatically de se. However, the four logically possible combinations of interpretations only give rise to three patterns of strict and sloppy identity. Consider first the case in which the first pronoun in the antecedent VP is interpreted as semantically de se. In this case it will uniformly give rise to a sloppy identity interpretation in the elided VP. If the second pronoun in the antecedent VP is interpreted semantically de se as well, it too will give rise to a sloppy identity reading, accounting for the sloppy-sloppy possibility. If the second pronoun in the antecedent VP is interpreted pragmatically de se instead, it will give rise to a strict identity reading in the elided VP as do all pragmatically de se pronouns, accounting for the sloppy-strict possibility. Consider now the case in which the first pronoun in the antecedent VP is given a pragmatically de se interpretation. In this case the corresponding pronoun in the elided VP will be uniformly given a strict identity interpretation. If the second pronoun in the antecedent $\mathrm{VP}$ is interpreted semantically de se, the reference of the corresponding pronoun in the elided VP will depend on its indirect antecedent in the elided VP. Since this antecedent is a pronoun which is interpreted strictly, the second elided pronoun will likewise be interpreted strictly, giving rise to a strict-strict interpretation. If the second pronoun in the elided VP is interpreted pragmatically de se, once again it will be given a strict interpretation thus giving a second means for generating a strictstrict interpretation. This leaves a total of three possible combinations of strict and sloppy identity readings - exactly the three listed in cell (f) of Table 2 .

\subsection{Explaining the Difficulties}

F\&M (1994) claimed that the VP-elided pronouns in examples like (3) do not have a strict-sloppy pattern of interpretation. However, such a pattern of interpretation can be generated on the above analysis by taking the antecedent pronouns to be as indicated in either of cells (b) or (e) of Table 2. Cell (b) is the case in which the first pronoun in the first sentence is interpreted as taking an antecedent outside of that sentence and the second pronoun is interpreted as de se with respect to the matrix subject. This interpretation is relatively easy to obtain, and 
straightforwardly gives rise to a strict-sloppy pattern of interpretation for the VP-elided pronouns. I assume that this fact was simply overlooked by $F \& M$, since they considered examples like those in (3) only in isolation, and hence implicitly excluded cases of extra-sentential anaphora from consideration. Cell (e), on the other hand, represents an interpretation that does not require any sentence external context. The fact that this interpretation is difficult to obtain can be taken as a partial explanation of why F\&M claim that a strict-sloppy reading of the VP-elided pronouns is impossible. In order for the analysis presented in this paper to be complete, it is necessary to determine the source of the difficulty in obtaining this interpretation.

The interpretation of the first sentence of (3b) represented in cell (e) of Table 2 is that in which both pronouns are interpreted de se and both take as their antecedent the matrix subject. For the first embedded pronoun, this is the only option available for generating a de se interpretation. For the second pronoun, however, a de se interpretation could equally well be generated by taking the first pronoun as its antecedent. I propose that it is the skipping of this potential antecedent which makes this interpretation difficult to obtain. Note, however, that skipping a potential antecedent in generating a de se interpretation for a pronoun is not in general problematic. For example, when the actual long distance antecedent and a non-actual but potential local antecedent have distinct referents, there is no problem in interpreting a pronoun as referring de se to the long distance antecedent. The pronoun he in (17), for example, can readily refer de se to Max despite the intervening potential antecedent $O$ scar.

(17) Max claims that Oscar swore he was innocent.

The problem of generating the interpretation indicated in cell (e) of Table 2 arises only in the special case in which the intervening potential antecedent is itself interpreted de se with respect to the long distance potential antecedent. Explaining why the interpretation in cell (e) of Table 2 is difficult can then be reduced to the problem of explaining why an intervening de se pronoun should block de se interpretation via a long distance coreferential antecedent.

To see why this should be the case, consider in more detail what de $s e$ interpretation of the pronouns amounts to. As outlined above, de se interpretation involves attribution of reference by an individual $x$ to that same individual $x$ via a thought taken to be one of $x$ 's own thoughts. For the first pronoun of $(3 b)$ this amounts to the speaker 
attributing to Max reference to himself as that individual who has some thought $t$ which is a directly experienced thought of Max's. Since Max's making a claim is his giving public expression to a private thought $T$, the thought expressed has to be one attributed to Max, and not simply a thought of the speaker's. The pronoun both characterizes the thought expressed (since $t$ is a constituent of $T$ ) and refers by way of a thought of Max's $(t)$, and so the thought expressed is understood to involve reference by Max to himself in just that special way immune to error through misidentification. This relation is illustrated in Diagram 1.

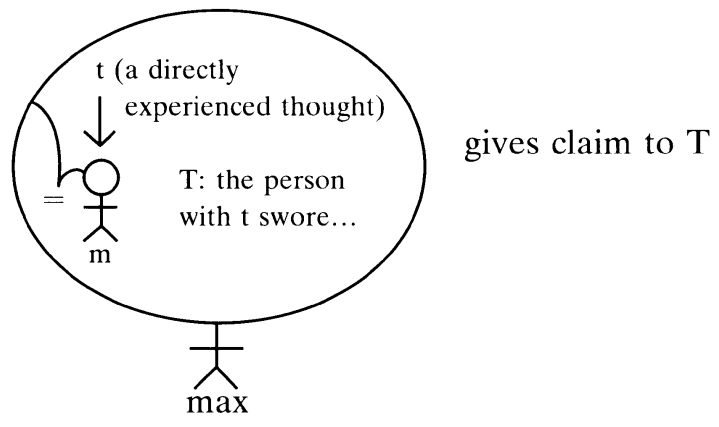

Diagram 1

In this diagram, Max is represented as representing himself as an individual, just as he would represent other people as individuals. The difference between his representation of himself and his representation of others is that only to the former does he attribute the thoughts he directly experiences. This attribution forms the basis for Max's identification of the represented individual $m$ with the experiencing individual max, represented above by the "=" sign.

What holds of the thought (I claim) Max gives claim to in uttering (3b) holds as well for the thought $T^{\prime}$ which according to (3b) Max claims he gave expression to as a swearing. (3b) can thus be represented in more detail as follows. 


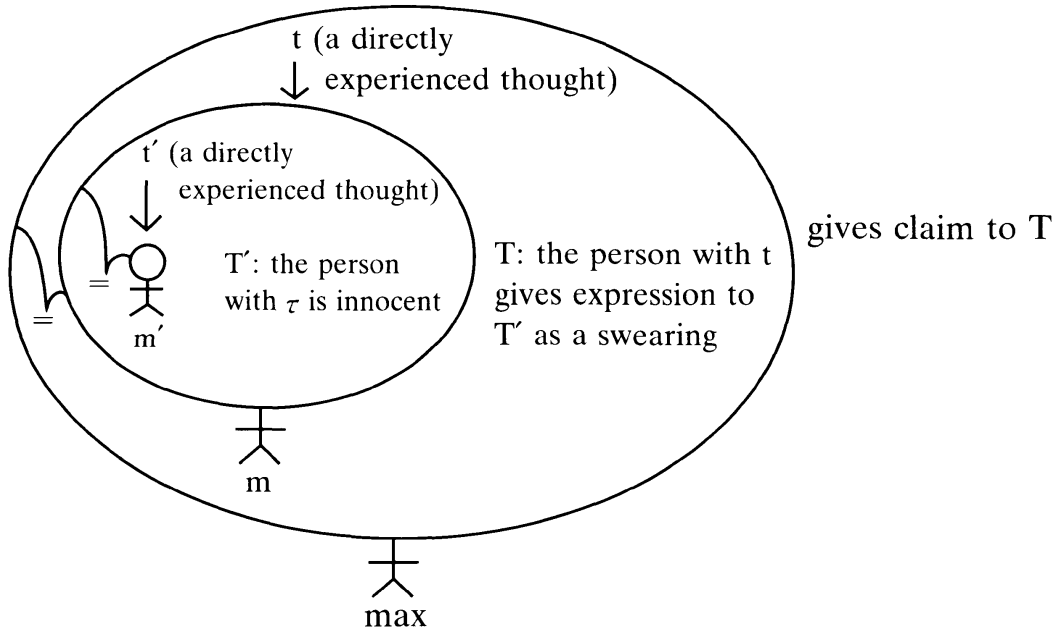

Diagram 2

In a normal case of attitude attribution, identification of $\tau$ in Diagram 2 with $\mathrm{t}^{\prime}$ would give rise to a local de se interpretation, and identification with $\mathrm{t}$ would give rise to a long distance de se interpretation. However, this distinction is blurred by the fact that the two represented individuals to whom "the person with $\tau$ " could refer, namely $m$ and $m^{\prime}$, are identified both with each other and with max as an experiencing individual. It is not unreasonable to assume that when faced with the problem of identifying $\tau$ in such a situation a normal speaker/hearer will adopt a strategy of least effort, opting for local identification whenever this is consistent with the referential connections the speaker intends to convey. ${ }^{11}$ This limits the possible value of $\tau$ to $t^{\prime} .{ }^{12}$ This strategy is best seen as a heuristic which is normally followed rather than as part of the mechanisms of interpretation which are automatical-

11 Note that this is consistent with the ready availability of the interpretations in cell (b) of Table 2, in which the first pronoun of $(3 \mathrm{~b})$ is interpreted as anaphoric outside of the sentence it occurs in. Though the speaker of (3b) makes an identification between the reference of the subject Max and the reference of the first pronoun he in this instance, the identification is not, or at least need not be, attributed to Max in this case.

12 Whether $t^{\prime}$ is in turn identified with $T^{\prime}$ is a separate question, one which will determine whether the de se reference in question is semantic or pragmatic. 
ly followed without exception. It is, after all, not impossible to interpret (3b) as in cell (e) of Table 2 if one puts enough effort into discerning the relevant interpretations. Not impossible, but difficult.

\subsection{Consequences of the Analysis}

The analysis of strict/sloppy identity patterns of VP-elided pronouns given above predicts that the availability of any given pattern depends not on the c-command relations that obtain between the referential expressions involved but rather on how the relevant pronouns can be interpreted. A sentence containing two VP-elided pronouns has four logically possible patterns of strict/sloppy identity interpretation. When one of the pronouns has the potential to be interpreted de se with respect to the other which in turn can be interpreted de se with respect to an expression outside the elided VP, the analysis above predicts that in the absence of cross-sentential anaphoric interpretation of the antecedent pronouns only three of these four logical possibilities will surface as readily available interpretations. This result is independent of the c-command relations that obtain among the relevant referring expressions, in contrast to the analysis of F\&M.

Support for this analysis comes from the behavior of psych predicates under VP ellipsis, as in (18).

(18) Max claims that his acne bothers him. Oscar does/claims that his acne bothers him too.

Sentences headed by a psych predicate appear to have a surface structure similar to that of their non-psych predicate counterparts, consisting of a subject, verb and object. However, as distinct from other predicates, their objects are interpreted as experiencers. On the present analysis this means that psych predicates attribute a thought to (the reference of) their objects which I assume must be about (the reference of) their subjects. This means that psych predicates should allow a reverse de se interpretation, one in which a pronoun embedded within the subject is interpreted de se with respect to the object, which in turn leads us to predict that the available patterns of interpretation should be the reverse of what we saw above. In particular, a strict-sloppy identity pattern should be readily available without any special context, generable when both pronouns are given a local de se interpretation, but a sloppy-strict pattern should be much more difficult. This prediction is borne out in (18) above: the second sentence in (18) can readily mean that Oscar claims that Max's acne bothers Oscar, but it is much 
more difficult to interpret the sentence to mean that Oscar claims that Oscar's acne bothers Max. The full pattern of judgments predicted to be available is given in Table 4 .

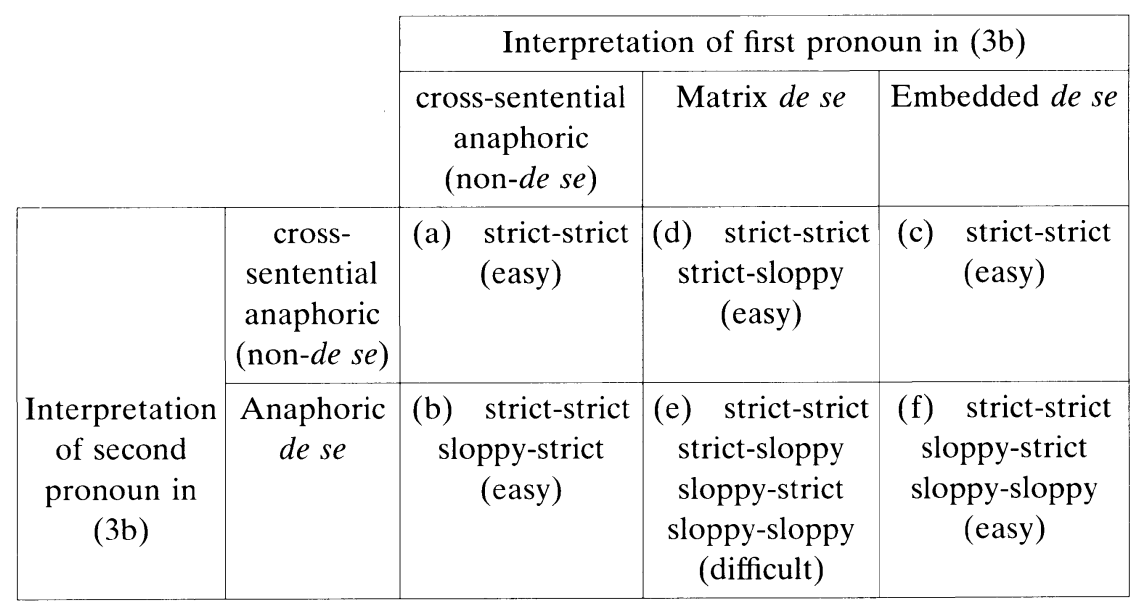

TABLE 4: Correlation between strict/sloppy identity and type of interpretation of the antecedent pronouns in (18).

I leave it to the reader to verify that these predictions are all borne out for the sentence in (18).

\subsection{Loose Ends}

The analysis developed above has several desirable consequences. However, there remains one potentially damaging objection. It was noted in (5) that non-de se intra-sententially anaphoric pronouns can give rise to either a strict identity or a sloppy identity interpretation in VP ellipsis, and that with multiple pronouns there is no restriction on the distribution of strict and sloppy interpretations. It is obvious, however, that the mechanisms used to generate strict and sloppy identity readings for de se pronouns cannot be used in these cases as well. In particular, sloppy identity readings cannot be generated by interpreting the pronouns as semantically de se since there is no thought attributed to the intended antecedent of the pronoun via which the pronouns could secure their reference. This means that some independent mechanism is required which can give rise to a 4 way ambiguity in the non-de se cases. The potential objection is that such mechanisms should be equally capable of applying to the pronouns that do have a 
potential de se interpretation as to those which don't, obviating the explanation given for the difficulty of obtaining a strict-sloppy identity reading for sentences like those in (3) considered out of context.

Though this objection cannot be dismissed lightly, it is by no means devastating. One need only assume that de se interpretation preferentially blocks non-de se interpretation. Though there are many ways of implementing this idea, I give only one here without justifying it as against alternatives.

If a pronoun $p$ can be de se anaphoric on an antecedent $a$, then $p$ cannot be non-de se anaphoric on $a$.

The upshot of this stipulation is that the examples in (3) cannot be analyzed in the same way as the examples in (5), whatever analysis one adopts for the latter.

\section{Conclusion}

The analysis developed in this paper is based on a semantic differentiation of distinct ways of determining the interpretations of pronouns. Significantly, accounting for the full range of possible interpretations available for elided VPs containing multiple occurrences of pronouns was accomplished without making any reference to syntactic mechanisms of encoding referential possibilities such as indices. Though the analysis rests on a partial rejection of F\&M's factual claims regarding the distribution of strict/sloppy interpretations for such sentences, it goes well beyond either F\&M's, Sag's or Williams' analyses of the phenomenon. These previous analyses fail to account for the relations that hold between de se interpretation, non-de se anaphoric interpretation and strict and sloppy identity. The analysis presented here not only accounts for these relations, but additionally gives a simple explanation for the relative difficulty of obtaining certain patterns of interpretation for VP-elided pronouns based upon them. These results were made possible by focusing not merely on the question of what the referent of a pronoun is but also on how this reference comes about. I hope that this shift of focus will help to bring us out of the dark ages of pronominal interpretation in which we have been subsisting for so long. 


\section{REFERENCES}

Castañeda, Hector-Neri (1966) "He: A Study in the Logic of Self-Consciousness," Ratio 7, 130-157.

Castañeda, Hector-Neri (1967a) "Indicators and Quasi-Indicators," American Philosophical Quarterly 4, no. 2, 85-100.

Castañeda, Hector-Neri (1967b) "On the Logic of Self-Knowledge" Nous 1, 9-22.

Castañeda, Hector-Neri (1968) "On the Logic of Attributions of Self-Knowledge to Others," Journal of Philosophy 65, no. 15, 439-456.

Chierchia, Gennaro (1989) "Anaphora and Attitudes de se," Language in Context, ed. by Renate Bartsch, Johan van Benthem and Peter van Emde Boas, 1-32, Foris, Dordrecht.

Chomsky, Noam (1995) The Minimalist Program, MIT Press, Cambridge, MA.

Chomsky, Noam (2000) "Minimalist Inquiries: The Framework," Step by Step: Essays on Minimalist Syntax in Honor of Howard Lasnik, ed. by Roger Martin, David Michaels and Juan Uriagereka, 89-155, MIT Press, Cambridge, MA.

Evans, Gareth (1982) The Varieties of Reference, Oxford University Press, Oxford.

Fiengo, Robert and Robert May (1994) Indices and Identity, MIT Press, Cambridge, MA.

Higginbotham, James (1980) "Pronouns and Bound Variables," Linguistic Inquiry $11,679-708$.

Higginbotham, James (1983) "Logical Form, Binding, and Nominals," Linguistic Inquiry, 14.3, 395-420.

Higginbotham, James (1985) "On Semantics," Linguistic Inquiry 16, 547-593.

Higginbotham, James (19891) "Belief and Logical Form," Mind and Language 6, 344-369.

Lewis, David (1979) "Attitudes de dicto and de se," The Philosophical Review 88, 513-543.

Oshima, Yoshikazu (2000) "Logophoricity and De Se Interpretation," Masters thesis, the University of Tokyo.

Reinhart, Tanya (1983) "Coreference and Bound Anaphora: A Restatement of the Anaphora Questions," Linguistics and Philosophy 6, 47-88.

Reinhart, Tanya (1986) "Center and Periphery in the Grammar of Anaphora," Studies in the Acquisition of Anaphora, Volume I, ed. by B. Lust, 123-150, Reidel, Dordrecht.

Sag, Ivan (1976a) Deletion and Logical Form, Doctoral dissertation, MIT.

Sag, Ivan (1976b) "A Logical Theory of Verb Phrase Deletion," CLS 12, 533-547.

Tancredi, Christopher (1992) Deletion, Deaccenting and Presupposition, Doctoral dissertation, MIT.

Tancredi, Christopher (1997) "Pronouns and Perspectives," Atomism and Bind- 
ing, ed. by Hans Bennis, Pierre Pica and Johan Rooryck, Foris, Dordrecht.

Tancredi, Christopher (2000) "Semantic Anaphora and Discourse Licensing: New Foundations for a Binding Theory," Proceedings of Nanzan GLOW, Nanzan University, Nagoya, Japan, 385-406.

Williams, Edwin (1977) "Discourse and Logical Form," Linguistic Inquiry 8, 101-139.

Graduate School of Arts and Sciences

University of Tokyo

3-8-1 Komaba, Meguro-ku

Tokyo 153-8902 